\title{
Diagnostic performance of FDG-PET/CT of post-transplant lymphoproliferative disorder and factors affecting diagnostic yield
}

\author{
F. M. Montes de Jesus ${ }^{1}$ (D) - T. C. Kwee ${ }^{2} \cdot$ X. U. Kahle ${ }^{3} \cdot$ M. Nijland $^{3} \cdot$ T. van Meerten ${ }^{3} \cdot$ G. Huls ${ }^{3} \cdot$ R. A. J. O. Dierckx ${ }^{1} \cdot$ \\ S. Rosati ${ }^{4}$ - A. Diepstra ${ }^{4}$-W. van der Bij ${ }^{5}$ - E. A. M. Verschuuren ${ }^{5}$ - A. W. J. M. Glaudemans ${ }^{1} \cdot$ W. Noordzij ${ }^{1}$
}

Received: 15 July 2019 / Accepted: 7 August 2019 / Published online: 24 August 2019

(C) The Author(s) 2019

\begin{abstract}
Purpose Post-transplant lymphoproliferative disorder (PTLD) is a serious complication after solid organ and hematopoietic stem cell transplantation, requiring a timely and accurate diagnosis. In this study, we evaluated the diagnostic performance of FDG$\mathrm{PET} / \mathrm{CT}$ in patients with suspected PTLD and examined if lactate dehydrogenase (LDH) levels, Epstein-Barr virus (EBV) load, or timing of FDG-PET/CT relate to detection performance of FDG-PET/CT.

Methods This retrospective study included 91 consecutive patients with clinical suspicion of PTLD and a total of 97 FDG-PET/CT scans within an 8-year period. Pathology reports and a 2-year follow-up were used as the reference standard. Diagnostic performance of FDG-PET/CT for detection of PTLD as well as logistic regression analysis for factors expected to affect diagnostic yield were assessed. Results The diagnosis of PTLD was established in 34 patients (35\%). Fifty-seven FDG-PET/CT scans (59\%) were true negative, $29(30 \%)$ were true positive, $6(6 \%)$ false positive, and $5(5 \%)$ false negative. Sensitivity of FDG-PET/CT for the detection of PTLD was $85 \%$, specificity $90 \%$, positive predictive value $83 \%$, and negative predictive value $92 \%$, with good inter-observer variability $(k=0.78)$. Of the parameters hypothesized to be associated with a true positive FDG-PET/CT result for the diagnosis of PTLD, only LDH was statistically significant (OR 1.03, $p=0.04$ ).

Conclusion FDG-PET/CT has a good diagnostic performance in patients suspected of PTLD, with a good inter-observer agreement. Only LDH levels seemed to influence the detection performance of FDG-PET/CT. EBV-DNA load and timing of FDG$\mathrm{PET} / \mathrm{CT}$ after transplantation did not affect FDG-PET/CT diagnostic yield.
\end{abstract}

Keywords Post-transplant lymphoproliferative disorder $\cdot{ }^{18}$ F-Fluoro-D-deoxyglucose positron emission tomography $\cdot$ FDG-PET/ $\mathrm{CT} \cdot$ Diagnosis

This article is part of the Topical Collection on Advanced Image Analyses (Radiomics and Artificial Intelligence)

F. M. Montes de Jesus

f.m.montes.de.jesus@umcg.nl

1 Department of Nuclear Medicine and Molecular Imaging, University of Groningen, University Medical Center Groningen, Hanzeplein 1, Groningen 9700 RB, The Netherlands

2 Department of Radiology, University of Groningen, University Medical Center Groningen, Groningen, The Netherlands

3 Department of Hematology, University of Groningen, University Medical Center Groningen, Groningen, The Netherlands

4 Department of Pathology and Medical Biology, University of Groningen, University Medical Center Groningen, Groningen, The Netherlands

5 Department of Pulmonary Diseases and tuberculosis, University of Groningen, University Medical Center Groningen,

Groningen, The Netherlands

\section{Introduction}

Post-transplant lymphoproliferative disorder (PTLD) is a serious complication after solid organ (SOT) and hematopoietic stem cell transplantation (HSCT). PTLD encompasses a heterogeneous morphologic spectrum, ranging from EBV driven polyclonal proliferations to aggressive monomorphic large $\mathrm{B}$ cell lymphomas. According to the World Health Organization, PTLD can be classified into 4 main groups: non-destructive lesions, polymorphic PTLD, monomorphic PTLD, and classical Hodgkin lymphoma PTLD [1].

PTLD has a variable clinical presentation that may include B-symptoms, lymphadenopathy, organ/allograft dysfunction, or a combination of non-specific symptoms. It is characterized by a bimodal presentation curve, with a peak incidence within 1 year after transplantation and a second peak after 4-5 years $[2,3]$. However, the diagnosis of PTLD can be made at any 
time after transplantation and diagnostic work-up is usually initiated by the presence of B-symptoms and biochemical anomalies such as an increase in lactate dehydrogenase (LDH) and detectable Epstein-Barr virus (EBV) DNA. In PTLD, elevated LDH has been associated with a lack of response to initial therapy and worse prognosis [4-6]. EBV is recognized to play a crucial role in the immunopathogenesis of PTLD and EBV-DNA load monitoring is routinely performed for early detection of PTLD [7]. However, LDH and EBV-DNA load are non-specific for the detection of PTLD and particularly EBV-DNA load is not useful in EBV-negative PTLD which may compromise up to $50 \%$ of PTLD cases [8-10]. During diagnostic work-up, ${ }^{18}$ F-fluoro-2-deoxy-Dglucose (FDG) positron emission tomography (PET)/computed tomography (CT) may also be performed, allowing for whole-body visualization of metabolic active lesions and direct biopsy localization.

Although FDG-PET/CT is an established imaging modality in the detection of other FDG-avid lymphomas, with a reported median sensitivity of $90 \%$ and specificity of $91 \%$, its diagnostic performance has not been extensively evaluated in PTLD [11, 12]. FDG-PET/CT may become an essential part of the diagnostic work-up in PTLD patients if proven to be of additional value for the detection of this disorder. However, biomarkers such as LDH, EBV-DNA load, and timing of FDG-PET/CT after transplantation may also influence its diagnostic yield. The purpose of this study was to evaluate the diagnostic performance of FDG-PET/CT in patients with suspected PTLD and to examine if LDH levels, EBV-DNA load, and timing after transplantation influence the detection performance of FDG-PET/CT.

\section{Materials and methods}

\section{Study design and patients}

This retrospective study included all consecutive patients between January 2010 and January 2019 for whom an FDG$\mathrm{PET} / \mathrm{CT}$ scan was requested. Indications for FDG-PET/CT requests are described in Table 1. The first FDG-PET/CT or in some patients the second scan after a 2-year negative follow-up period was included in the analysis (see "Reference standard"). Only patients 19 years and older were included. Patients with central nervous system involvement, complete tumor resection prior to FDG-PET/CT evaluation, and those without a biopsy or 2-year follow-up were excluded.

\section{Patient record review}

Relevant clinical and biochemical data were collected from the electronic patient files at the University Medical Center Groningen. These included age, gender, organ transplanted, time between transplantation and FDG-PET/CT, LDH levels, EBV-DNA load, and PTLD morphology and histology.

\section{FDG-PET/CT acquisition and interpretation}

All FDG-PET/CT scans were performed on a Siemens Biograph 40 or 64 slice mCT (Siemens Healthineers, Erlangen, Germany) according to the European Association of Nuclear Medicine (EANM) procedure guidelines for tumor imaging [13]. Scans were performed after a minimum fasting time of $6 \mathrm{~h}$. Images from the mid-thigh to skull base were acquired $60 \mathrm{~min}$ after intravenous administration of $3 \mathrm{MBq} /$ kg FDG. Integrated FDG-PET/CT images were corrected for scatter and attenuation based on CT information. Scans were retrospectively reviewed by 3 readers ( 2 experienced nuclear medicine physicians (AG and $\mathrm{WN}$ ) and 1 research fellow (FMJ) using syngo.via software (Siemens Healthineers, Erlangen, Germany). Readers reviewed the scans independently from each other and were blinded for other clinical/ imaging findings and pathology results. Scans were considered positive for PTLD when FDG-avid lesions were present that could not be related to other pathology than PTLD. Scans were considered negative when no FDG-avid lesions suspicious for PTLD were found. Scans were considered equivocal when FDG-avid lesions were present, but this uptake could be due to either PTLD or due to other diseases/malignancy. In these cases, a differential diagnosis was noted. Discordant results between readers were re-evaluated in a consensus
Table 1 Indications for FDG$\mathrm{PET} / \mathrm{CT}$ request*

\begin{tabular}{lr}
\hline & $\mathrm{N}(\%)$ \\
\hline $\begin{array}{l}\text { Blood panel disturbances } \\
\text { (e.g., complete blood count and biochemistry) }\end{array}$ & $20(20.6)$ \\
High EBV-DNA load & 44 (45.3) \\
Physical symptoms & $37(38.1)$ \\
(e.g., B-symptoms, enlarged lymph nodes, other non-specific symptoms) & $38(39.2)$ \\
Anomalies previous examination & \\
(e.g., colonoscopy, other non FDG-PET/CT imaging) & \\
\hline
\end{tabular}

*Multiple indications possible for a single scan 
meeting and conclusively classified as positive or negative for PTLD.

\section{Reference standard}

Pathology reports were used as a reference standard for PTLD diagnosis. Two experienced hematopathologists (SR, AD) were consulted for morphology clarification when necessary. In case of a PTLD-negative biopsy or lack of tissue for pathological examination, a 2-year follow-up period without pre-emptive PTLD therapy was accepted as the reference standard. Absence of lymphoma during this period has been shown to be an accurate marker for lack of disease in other lymphomas $[14,15]$. True positive cases were defined as a PTLDpositive FDG-PET/CT and a PTLD-positive pathology result within 2 years after FDG-PET/CT. True negative cases were defined as a PTLD-negative FDG-PET/CT and no signs of PTLD within 2-year follow-up. False positive cases were defined as a PTLD-positive FDGPET/CT and no signs of PTLD within a 2-year followup. False negative results were defined as a PTLDnegative FDG-PET/CT and pathology proven PTLD within a 2-year follow-up period.

\section{Statistical analysis}

Baseline patient characteristics were summarized using medians with interquartile range (IQR) for nonnormally distributed variables. Sensitivity, specificity, positive predictive value (PPV), and negative predictive value (NPV) of FDG-PET/CT for the detection of PTLD were calculated with a $95 \%$ confidence interval (CI). Logistic regression model analysis was carried out using mean serum LDH levels, mean EBV-DNA load (mean over a 31-day period before FDG-PET/CT), and time between transplantation and FDG-PET/CT with FDG-PET/CT result as a dependent variable. FDG$\mathrm{PET} / \mathrm{CT}$ results were dichotomized as true positive or not true positive (i.e., false positive, true negative, and false negative combined). The corresponding odds ratio (OR) and 95\% CI were calculated. Statistical significance was set at $p$ value $\leq 0.05$. Inter-observer variability between the 3 observers was calculated using Fleiss kappa. The kappa value was interpreted according to the method of Landis and Koch: poor (0 to 0.20$)$, fair $(0.21$ to 0.40$)$, moderate $(0.41$ to 0.60$)$, good $(0.61$ to 0.80$)$, and perfect agreement (0.81 to 1$)$ [16]. All statistical analyses were performed using SPSS, version 23.0 (IBM Corporation, Armonk, NY, USA).

\section{Results}

\section{Patients}

One-hundred-twelve potentially eligible patients were identified. Eleven patients were excluded due to central nervous system involvement, 6 due to complete tumor resection prior to FDG-PET/CT evaluation, and 4 due to lack of a reference standard. In total, 91 patients and 97 FDG-PET/CT scans were included in this study (Table 2). In 6 patients, 2 FDG-PET/CT scans were included because of PTLD suspicion on two different occasions with a time interval of more than 2 years. There were 50 males $(55 \%)$ and 41 females $(45 \%)$ with a median age of 54 years. The most frequently transplanted organ was the lung $(n=40,44 \%)$ followed by the kidney $(n=31,34.1 \%)$, liver $(n=11,12.1 \%)$, HSCT $(n=4,4.4 \%)$, multi-organ $(n=4,4.4 \%)$, and the heart $(n=1,1.1 \%)$. According to the reference standard, 34 patients (35\%) were diagnosed with PTLD. There were 21 EBV-positive PTLDs (62\%) and 13 EBV-negative PTLDs (38\%). The median time

Table 2 Patient characteristics $(n=91)$

\begin{tabular}{|c|c|}
\hline \multicolumn{2}{|l|}{ Age at diagnosis (years) } \\
\hline Median & 54 \\
\hline Range & $19-80$ \\
\hline IQR & 25 \\
\hline \multicolumn{2}{|l|}{ Gender } \\
\hline Male & $50(55 \%)$ \\
\hline Female & $41(45 \%)$ \\
\hline \multicolumn{2}{|l|}{ Transplanted organ } \\
\hline Lung & $40(44.0 \%)$ \\
\hline Kidney & $31(34.1 \%)$ \\
\hline Liver & $11(12.1 \%)$ \\
\hline HSCT & $4(4.4 \%)$ \\
\hline Multi-organ & $4(4.4 \%)$ \\
\hline Heart & $1(1.1 \%)$ \\
\hline \multicolumn{2}{|l|}{ Histology } \\
\hline Non-destructive & $2(5.9 \%)$ \\
\hline Polymorphic & $6(17.6 \%)$ \\
\hline Monomorphic & $24(70.6 \%)$ \\
\hline Classic Hodgkin type & $1(2.9 \%)$ \\
\hline Unclear & $1(2.9 \%)$ \\
\hline \multicolumn{2}{|l|}{ EBV status tumor } \\
\hline Positive & $21(62 \%)$ \\
\hline Negative & $13(38 \%)$ \\
\hline \multicolumn{2}{|c|}{ Time between transplant and FDG-PET/CT (years) } \\
\hline Median & 5 \\
\hline Range & $0-28$ \\
\hline IQR & 9 \\
\hline
\end{tabular}


between transplantation and FDG-PET/CT was 5 years (IQR; 9).

\section{Diagnostic performance of FDG-PET/CT}

After a consensus meeting, the three readers assessed 35 scans to be positive for PTLD and 62 scans negative. Pathology confirmation was the reference standard in 64 scans $(66 \%)$ and 2-year follow-up in $33(34 \%)$. According to the reference standard, 57 scans (59\%) were true negative, 29 (30\%) were true positive, while $6(6 \%)$ false positive, and $5(5 \%)$ false negative results were observed (Table 3 , Fig. 1). On a patient-based analysis, sensitivity of FDG-PET/CT for the detection of PTLD was $85 \%$, specificity $90 \%$, PPV $83 \%$, and NPV 92\% (Table 4).

\section{False positive and false negative scans}

In total, 6 scans were found to be false positive (Fig. 2). The final diagnoses of these false positive scans were as follows: 1 case of condyloma acuminata in the recto-uterine pouch, 1 case of an adenomatoid tumor in the round ligament, 1 case of small cell carcinoma in the transplanted lung, 1 case of systemic Nocardia infection, 1 case of Aspergillus infection in the lungs, and 1 case of spontaneous recovery of the suspected lesions without medical intervention, considered very unlikely to be PTLD. False positive results could be divided into two main categories: (1) other malignancies also showing high FDG uptake, making the differentiation between PTLD and other malignancy difficult and (2) infections, also taking up FDG and leading to a differential diagnosis of PTLD or infection. Five scans were concluded to be false negative: 2 cases with focal uptake in the tonsils/adenoid, interpreted as physiological uptake but confirmed to be nondestructive PTLD, 1 case with focal uptake in the rectum, interpreted as physiological uptake but confirmed to be polymorphic PTLD, 1 case with uptake in the lungs, interpreted as a primary lung tumor or infection but confirmed to be classic Hodgkin type PTLD, and 1 case considered to be a lung infiltrate without FDG-uptake but confirmed to be monomorphic PTLD (Table 5).

Table 3 Classification of FDG-PET/CT scans $(n=97)$

\begin{tabular}{lll}
\hline & $\begin{array}{l}\text { PTLD present } \\
\text { No. }(\%)\end{array}$ & $\begin{array}{l}\text { PTLD absent } \\
\text { No. }(\%)\end{array}$ \\
\hline PET positive & $29(29.9)$ & $6(6.2)$ \\
PET negative & $5(5.1)$ & $57(58.8)$ \\
\hline
\end{tabular}

\section{Determinants of detection performance of FDG-PET/CT}

According to univariate logistic regression, the only statistically significant parameter associated with a true positive FDG-PET/CT scan was serum LDH level with an OR of 1.03 ( $p=0.04,95 \%$ CI;1.001-1.06). Hence, for each 10 unit increase in LDH, the odds of having a true positive FDG-PET/ CT scan were $3 \%$ higher. The remaining parameters, EBVDNA load (OR; $1.0, p=0.59,95 \% \mathrm{CI} ; 1.00-1.00)$, and time between transplant and FDG-PET/CT (OR; $1.05, p=0.23$, 95\% CI;0.97-1.12) were not statistically significant in the univariate logistic regression analysis. For this reason, a multivariate logistic regression analysis was not performed (Table 6). Sub-analysis of only EBV-positive PTLD patients also revealed statistically insignificant results (OR; 1.0, $p=$ $0.64,95 \%$ CI; $1.00-1.00$ ).

\section{Inter-observer variability}

From a total of 97 FDG-PET/CT scans evaluated prior to consensus, discordant results were reported in 14 scans. The majority of discordant results were due to FDG uptake in the lung parenchyma $(n=7)$. The differential diagnosis for these lesions included PTLD, primary lung tumor or infection. There were 5 cases of discordant results observed in the gastrointestinal tract and 1 discordant result for lesions in the pelvic area, in which observers reported difficulty in distinguishing between pathological and physiological uptake. Finally, 1 discordant finding, located in the adenoids of a young patient, was difficult to characterize as either pathological or physiological/infectious. From the 14 discordant FDG-PET/CT scans, 2 were false positive and 3 were false negative. The inter-observer variability was found to be good at $k=0.78(95 \% \mathrm{CI} ; 0.68-0.88)$.

\section{Discussion}

Although metabolic imaging with FDG-PET/CT has an established role in the diagnosis of non-Hodgkin and Hodgkin lymphoma, few studies have been carried out to assert its detection performance in PTLD [11, 17]. In our study population, compromising of $97 \mathrm{FDG}-\mathrm{PET} / \mathrm{CT}$ scans in 91 patients with suspected PTLD, we found a sensitivity of $85 \%$, specificity of $90 \%$, PPV of $83 \%$, and NPV of $92 \%$ with good inter-observer variability $(k=0.78)$. Of the determinants hypothesized to influence detection performance of FDGPET/CT, only LDH levels were statistically significant with an OR of 1.03 ( $p=0.04,95 \%$ CI;1.001-1.06).

Current guidelines from the British Committee for Standards in Hematology, the British Transplantation Society, and National Comprehensive Cancer Network 
Fig. 1 A 49-year-old male presented with low LDH levels (251 U/l) and low EBV DNA (1010 copies $/ \mathrm{ml}$ ) load, 6 years after lung transplantation. FDG$\mathrm{PET} / \mathrm{CT}$ was requested after palpable lymphadenopathy was clinically detected. Maximum intensity projection FDG-PET image shows metabolically active supraclavicular and mediastinal lymph nodes, and a large confluent abdominal lesion. Axial fused $\mathrm{PET} / \mathrm{CT}$ (top right) and CT (bottom right) show the metabolically active supraclavicular lymph node, which proved to be monomorphic PTLD after biopsy

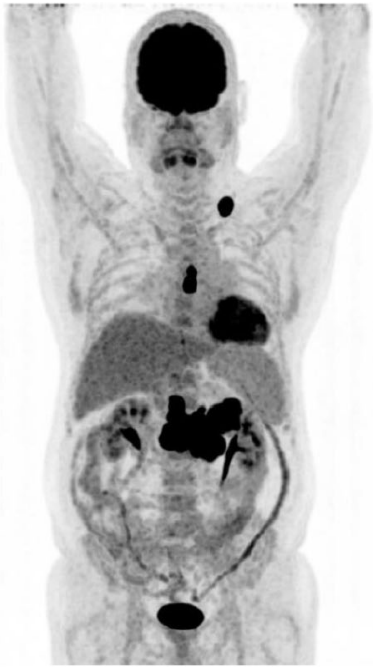

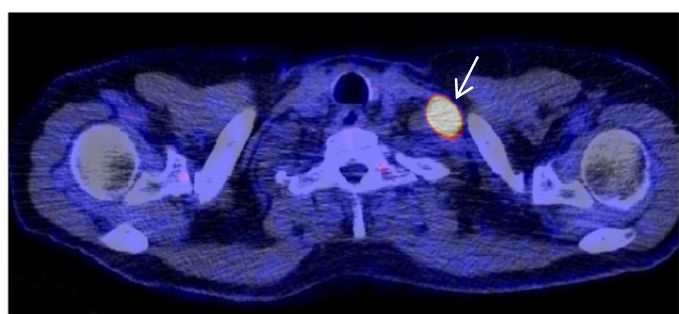

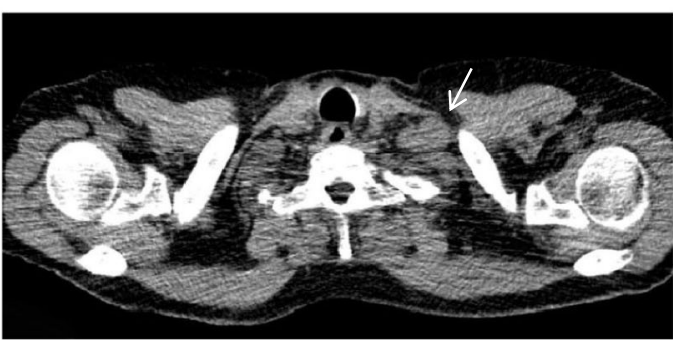

include no concrete recommendations on the use of FDGPET/CT for diagnosing PTLD $[18,19]$. The good diagnostic performance demonstrated in this study indicates that FDG$\mathrm{PET} / \mathrm{CT}$ is a valuable imaging modality for detecting PTLD. The good inter-observer variability also indicates that FDGPET/CT interpretation was minimally reader dependent between two nuclear physicians and a junior reader. We identified common limitations associated with FDG-PET/CT false positive results such as inflammatory conditions and other malignancies in addition to false negative results in nondestructive and polymorphic PTLD, specific to this patient population. Because non-destructive lesions are commonly found in Waldeyer's ring, such lesion location may be prone to be interpreted as physiological uptake $[20,21]$.

Although the majority of studies published on the diagnostic performance of FDG-PET/CT in PTLD are limited to case series, studies by Panagiotidis et al. and Dierickx et al. also reported good FDG-PET/CT diagnostic performance [22, 23$]$. Panagiotidis et al. included 40 patients with suspected PTLD and compared FDG-PET/CT diagnostic performance with CT. They concluded that FDG-PET/CT plays a significant role in the diagnosis of PTLD with high detection accuracy (sensitivity $88 \%$, specificity $91 \%$, PPV $88 \%$, and NPV 91\%) [22]. Some limitations of the study by Panagiotidis et al. included a smaller study population, half of that included in our study, and FDG-PET/CT scans were evaluated by a nuclear

Table 4 Detection performance of FDG-PET/CT in PTLD

\begin{tabular}{lll}
\hline Analysis & Value \% & $95 \%$ CI \\
\hline Sensitivity & 85 & $68-94$ \\
Specificity & 90 & $80-96$ \\
Positive predictive value & 83 & $66-93$ \\
Negative predictive value & 92 & $81-97$ \\
Accuracy & 89 & $81-94$ \\
\hline
\end{tabular}

medicine physician and a radiologist without blinding for clinical data. Dierickx et al. investigated FDG-PET/CT diagnostic performance in 125 cases and reported high sensitivity of FDG-PET/CT in detecting PTLD (sensitivity 90\%, specificity 89\%, PPV 85\%, and NPV 93\%) [23]. However, their study was performed between 2003 and 2010 with $43 \%$ of the study population scanned with a stand-alone PET system and no information on the duration of follow-up in negative cases. A recent systematic review on the clinical performance of different imaging modalities in the diagnosis and treatment response evaluation of PTLD patients reported FDG-PET(/ $\mathrm{CT})$ to be the most frequently used imaging modality and a promising tool in this setting [24]. In the same review, false positive results were reported due to inflammatory conditions while false negative results occurred in areas of high physiological background activity and in non-destructive PTLD lesions. These results not only indicate the utility of FDG-PET/ CT in PTLD patients but also concur with some of the findings in this study regarding potential causes for false positive and false negative results.

From the parameters hypothesized to be associated with a true positive FDG-PET/CT result for the diagnosis of PTLD, only LDH had a statistically significant odds increase. Uncontrolled proliferation of malignant cells with high cellular turnover is characterized by increased glycolysis and LDH release. High energy metabolism may translate into higher FDG uptake in tumor tissue and seemingly increase FDGPET/CT detection performance [25]. Nevertheless, the influence of LDH levels on the detection performance of FDG$\mathrm{PET} / \mathrm{CT}$ should be confirmed in future research given the fact that the lower boundary of the $95 \%$ CI of the OR was close to 1 in the present study. The role of EBV in the pathogenesis of PTLD is well documented; however, EBV-DNA load was not associated with true positive FDG-PET/CT results in our study [26]. EBV monitoring is a common clinical practice for early PTLD detection and various studies have advocated 
Fig. 2 A 62-year-old male in which elevated LDH levels (347 U/1) and EBV-DNA load $(1,032,500$ copies $/ \mathrm{ml})$ were found after clinical monitoring within 1 year after kidney transplantation. FDG-PET/CT was subsequently requested. Maximum intensity projection FDG-PET shows disseminated metabolically active cervical, mediastinal, and lung parenchymal lesions with focal pararenal, native kidney, mesenteric, and liver lesions. Axial fused FDG-PET/CT (top right) and CT (bottom right) show a metabolically active supraclavicular lymph node that proved to be a granulomatous inflammation due to a mycobacterium after biopsy
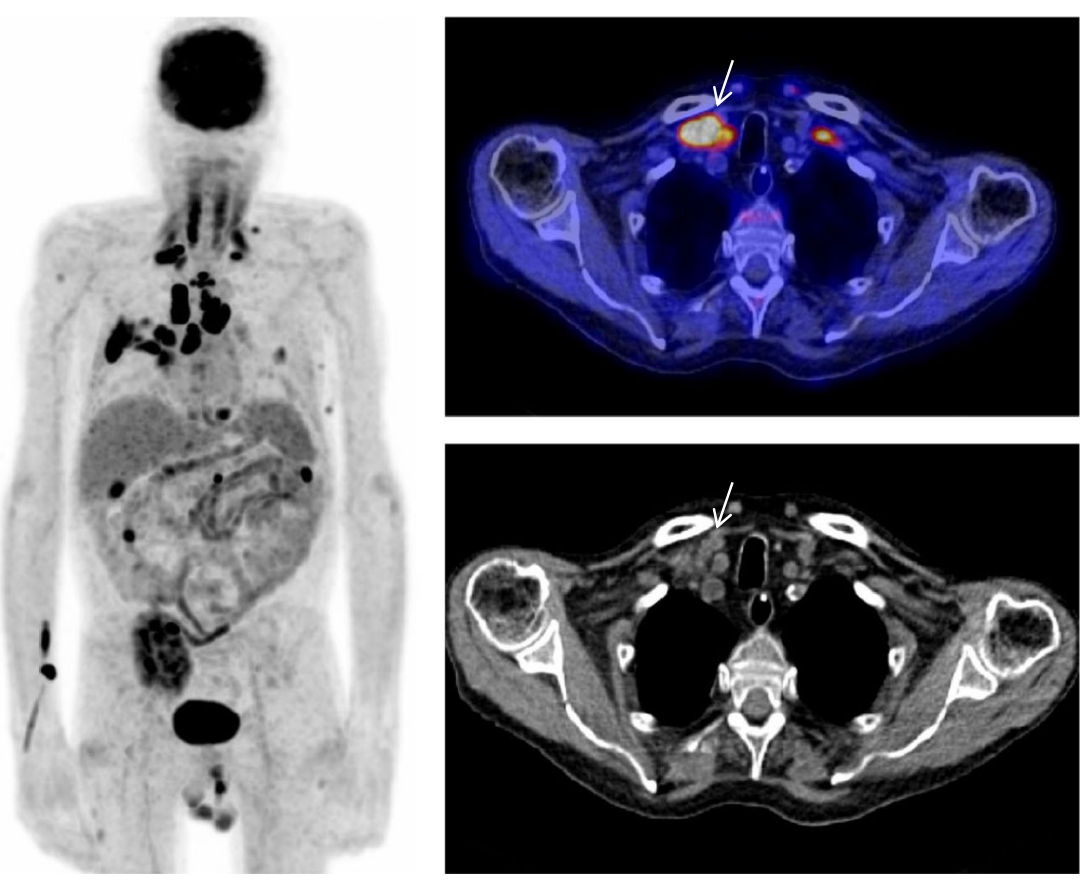

its importance as a predictor of PTLD development [3, 27-31]. Nonetheless, EBV-DNA load does not seem to affect the detection performance of FDG-PET/CT. One potential explanation is the high percentage of EBV-negative PTLD in our study population, which may have decreased the clinical utility of the EBV-DNA load covariate. Yet, in a subanalysis of only EBV-positive PTLD patients, we could not demonstrate a relationship between EBV DNA load and a true positive FDG-PET/CT either. We hypothesize that a single time cutoff value may not be indicative of imminent PTLD and consequently the need for FDG-PET/CT scanning.
Instead, changes over time in EBV DNA load may yield more clinically relevant information as proposed by other studies $[32,33]$.

Due to the retrospective design of this study, information on immunosuppression adjustments was not available. As a consequence, it was not possible to evaluate EBV-DNA load changes over time as this is affected by immunosuppression intensity. PTLD patient population is inherently heterogeneous with regard to medical history, immunosuppression regimens, and treatment approaches. In our retrospective study, heterogeneity might have been introduced by the 8 -year

Table 5 Description false positive/negative cases

\begin{tabular}{|c|c|c|c|}
\hline & Readers' differential diagnosis* & Location FDG uptake & Final diagnosis/outcome \\
\hline \multirow[t]{10}{*}{ False positive $(n=6)$} & PTLD & Recto-uterine pouch & Condyloma acuminata \\
\hline & Pelvic malignancy & & \\
\hline & PTLD & $\begin{array}{l}\text { Round ligament, intra-abdominal, } \\
\text { retroperitoneal and inguinal lymph nodes }\end{array}$ & Adenomatoid tumor \\
\hline & PTLD & Cervical, retroperitoneal lymph nodes, liver & Spontaneous recovery \\
\hline & Liver abscess & & \\
\hline & PTLD & Supraclavicular, mediastinal, hilar and & Systemic (Nocardia) infection \\
\hline & Disseminated infection & mediastinal lymph nodes, lung & \\
\hline & PTLD & Lung & Small cell carcinoma \\
\hline & Lung malignancy & & \\
\hline & PTLD & Mediastinal lymph nodes, lung & Aspergillus infection \\
\hline \multirow[t]{7}{*}{ False negative $(n=5)$} & $\begin{array}{l}\text { Inflammation } \\
\text { PTLD }\end{array}$ & Tonsils & Non-destructive PTLD tonsils \\
\hline & Pneumonia & Lung & Classic non-Hodgkin PTLD lung \\
\hline & Lung malignancy & & \\
\hline & PTLD & & \\
\hline & Physiologic uptake & Tonsils, adenoids & Non-destructive PTLD tonsils \\
\hline & Physiologic uptake & Pelvis & Polymorphic PTLD rectum \\
\hline & Unspecific lung infiltrate & No uptake & Monomorphic PTLD lung \\
\hline
\end{tabular}

*In order of most likely diagnosis 
Table 6 Association of parameters with a true positive FDG-PET/CT result for the diagnosis of PTLD

\begin{tabular}{llrl}
\hline Parameter & Univariate OR & $95 \%$ CI & $p$ \\
\hline Serum LDH levels & 1.03 & $1.001-1.06$ & 0.04 \\
EBV-DNA load & 1.00 & $1.00-1.00$ & 0.59 \\
EBV-DNA load (EBV-positive PTLD cases) & 1.00 & $1.00-1.00$ & 0.64 \\
Time between transplant and FDG-PET/CT & 1.05 & $0.97-1.12$ & 0.23 \\
\hline
\end{tabular}

period inclusion time within various medical departments. Lack of standardization limits the analysis on how patient selection and timing of FDG-PET/CT affect its diagnostic performance, potentially inducing selection bias. Likewise, due to the retrospective nature of this study, we were not able to evaluate how different immunosuppressive regimens might have affected PTLD pathogenesis, which is of great clinical relevance and should be considered in future studies. Despite these limitations, the data presented reflects current clinical practice, as immunosuppression regimens are often department specific and there are currently no guidelines on the use FDG-PET/CT for the diagnosis of PTLD. Additional future studies may also focus on the role of interim and end-oftreatment FDG-PET/CT on survival, already explored by Keerberghen et al. and Zimmermann et al., and on the role of FDG-PET/CT in pediatric PTLD patients [34, 35]. Considering the marked differences in incidence, (imaging) presentation, and prognosis of the disease between children and adults, pediatric specific studies are warranted.

\section{Conclusion}

FDG-PET/CT has a good diagnostic performance in patients with suspected PTLD. False positive results were due to other malignancies or infections while false negative results occurred in cases interpreted as physiological uptake (adenoids and rectum), other malignancy, and in 1 case of non-FDG-avid PTLD. Furthermore, a good inter-observer agreement was found, which further underlines the clinical utility of FDG-PET/CT. Only LDH levels seemed to influence the detection performance of FDG-PET/CT, while EBV-DNA load and time between transplant and FDG-PET/CT did not. The results of this study may help to implement FDG-PET/CT in future PTLD guidelines.

Author contributions All authors contributed to final analysis and writing of the manuscript.

Data availability The datasets used and/or analyzed during the current study are available from the corresponding author on reasonable request.

\section{Compliance with ethical standards}

Conflict of interests All authors declare that they have no conflict of interest.
Ethical approval All procedures performed were in accordance with the ethical standards of the institutional research committee and with the 1964 Declaration of Helsinki and its later amendments.

Informed consent According to the Dutch Medical Research Involving Human Subject Act, the local medical ethical committee of the University Medical Center Groningen exempted approval without additional procedures. No additional informed consent was required for this retrospective study.

Abbreviations CI, confidence interval; CT, computed tomography; EANM, European Association of Nuclear Medicine; EBV, Epstein-Barr virus; FDG, ${ }^{18}$ F-fluoro-2-deoxy-D-glucose; HSCT, hematopoietic stem cell transplantation; IQR, interquartile range; $\mathrm{LDH}$, lactate dehydrogenase; NPV, negative predictive value; OR, odds ratio; PET, positron emission tomography; PPV, positive predictive value; PTLD, post-transplant lymphoproliferative disorder; SOT, solid organ transplantation

Open Access This article is distributed under the terms of the Creative Commons Attribution 4.0 International License (http:// creativecommons.org/licenses/by/4.0/), which permits unrestricted use, distribution, and reproduction in any medium, provided you give appropriate credit to the original author(s) and the source, provide a link to the Creative Commons license, and indicate if changes were made.

\section{References}

1. Swerdlow SH, Campo E, Harris NL, Jaffe ES, Pileri SA, Stein H, et al. Post-transplant lymphoproliferative disorders (PTLD). In: Swerdlow S, Campo E, Harris N, Jaffe E, Pileri S, Stein H, et al., editors. World Health Organization Calssification of Tumours of Haematopoietic and Lymphoid Tissues, vol. 2017. 4th, revised ed. Lyon: International Agency for Research on Cancer; 2017. p. 45362.

2. Végso G, Hajdu M, Sebestyén A. Lymphoproliferative disorders after solid organ transplantation-classification, incidence, risk factors, early detection and treatment options. Pathol Oncol Res. 2011;17(3):443-54.

3. Bakker NA, van Imhoff GW, Verschuuren EAM, van Son WJ. Presentation and early detection of post-transplant lymphoproliferative disorder after solid organ transplantation. Transpl Int. 2007;20(3):207-18.

4. Dierickx D, Tousseyn T, Sagaert X, Fieuws S, Wlodarska I, Morscio J, et al. Single-center analysis of biopsy-confirmed posttransplant lymphoproliferative disorder: incidence, clinicopathological characteristics and prognostic factors. Leuk Lymphoma. 2013;54(11):2433-40.

5. Evens AM, David KA, Helenowski I, Nelson B, Kaufman D, Kircher SM, et al. Multicenter analysis of 80 solid organ transplantation recipients with post-transplantation lymphoproliferative disease: outcomes and prognostic factors in the modern era. J Clin Oncol. 2010;28(6):1038-46. 
6. Caillard S, Porcher R, Provot F, Dantal J, Choquet S, Durrbach A, et al. Post-transplantation lymphoproliferative disorder after kidney transplantation: report of a nationwide French registry and the development of a new prognostic score. J Clin Oncol. 2013;31(10): 1302-9.

7. Wareham NE, Mocroft A, Sengeløv H, Da Cunha-Bang C, Gustafsson F, Heilmann C, et al. The value of EBV DNA in early detection of post-transplant lymphoproliferative disorders among solid organ and hematopoietic stem cell transplant recipients. J Cancer Res Clin Oncol. 2018;144(8):1569-80.

8. Blaes AH, Morrison VA. Post-transplant lymphoproliferative disorders following solid-organ transplantation. Expert Rev Hematol. 2010;3(1):35-44.

9. Trappe R, Oertel S, Leblond V, Mollee P, Sender M, Reinke P, et al. Sequential treatment with rituximab followed by CHOP chemotherapy in adult B-cell post-transplant lymphoproliferative disorder (PTLD): the prospective international multicentre phase 2 PTLD1 trial. Lancet Oncol. 2012;13(2):196-206.

10. Nijland ML, Kersten MJ, Pals ST, Bemelman FJ, ten Berge IJM. Epstein-Barr virus-positive posttransplant lymphoproliferative disease after solid organ transplantation. Transplant Direct. 2016;2(1): e48.

11. Cheson BD. Staging and response assessment in lymphomas: the new Lugano classification. Chin Clin Oncol. 2015;4(1):1-9.

12. Isasi CR, Lu P, Blaufox MD. A metaanalysis of18F-2-deoxy-2fluoro-D-glucose positron emission tomography in the staging and restaging of patients with lymphoma. Cancer. 2005;104(5): 1066-74.

13. Boellaard R, Tatsch K, Pike LC, Testanera G, Kotzerke J, Graham $\mathrm{MM}$, et al. FDG PET/CT: EANM procedure guidelines for tumour imaging: version 2.0. Eur J Nucl Med Mol Imaging. 2014;42(2): 328-54.

14. El-Galaly TC, Jakobsen LH, Hutchings M, de Nully Brown P, Nilsson-Ehle H, Székely E, et al. Routine imaging for diffuse large B-cell lymphoma in first complete remission does not improve post-treatment survival: a Danish-Swedish population-based study. J Clin Oncol. 2015;33(34):3993-8.

15. Maurer MJ, Ghesquières H, Jais JP, Witzig TE, Haioun C, Thompson CA, et al. Event-free survival at 24 months is a robust end point for disease-related outcome in diffuse large B-cell lymphoma treated with immunochemotherapy. J Clin Oncol. 2014;32(10): 1066-73.

16. Landis JR, Koch GG. The measurement of observer agreement for categorical data. Biometrics. 1977;33(1):159.

17. El-Galaly TC, Gormsen LC, Hutchings M. PET/CT for staging; past, present, and future. Semin Nucl Med. 2018;48(1):4-16.

18. Parker A, Bowles K, Bradley JA, Emery V, Featherstone C, Gupte $\mathrm{G}$, et al. Diagnosis of post-transplant lymphoproliferative disorder in solid organ transplant recipients - BCSH and BTS guidelines. $\mathrm{Br}$ J Haematol. 2010;149(5):675-92.

19. National Comprehensive Cancer Network. Post-transplant lymphoproliferative disorders (version 2.2015). 2015.

20. Nelson BP, Wolniak KL, Evens A, Chenn A, Maddalozzo J, Proytcheva M. Early posttransplant lymphoproliferative disease. Am J Clin Pathol. 2012;138(4):568-78.

21. Scarsbrook AF, Warakaulle DR, Dattani M, Traill Z. Posttransplantation lymphoproliferative disorder: the spectrum of imaging appearances. Clin Radiol. 2005;60(1):47-55.

22. Panagiotidis E, Quigley A-M, Pencharz D, Ardeshna K, Syed R, Sajjan R, et al. (18)F-fluorodeoxyglucose positron emission tomography/computed tomography in diagnosis of post-transplant lymphoproliferative disorder. Leuk Lymphoma. 2014;55(3):515-9.

23. Dierickx D, Tousseyn T, Requilé A, Verscuren R, Sagaert X, Morscio J, et al. The accuracy of positron emission tomography in the detection of posttransplant lymphoproliferative disorder. Haematologica. 2013;98(5):771-5.

24. Montes de Jesus FM, Kwee TC, Nijland M, Kahle XU, Huls G, Dierckx RAJO, et al. Performance of advanced imaging modalities at diagnosis and treatment response evaluation of patients with posttransplant lymphoproliferative disorder: a systematic review and meta-analysis. Crit Rev Oncol Hematol. 2018;132(June):27-38.

25. Hanahan D, Weinberg RA. Hallmarks of cancer: the next generation. Cell. 2011;144(5):646-74.

26. Morscio J, Dierickx D, Tousseyn T. Molecular pathogenesis of Bcell posttransplant lymphoproliferative disorder: what do we know so far? Clin Dev Immunol. 2013;2013:150835.

27. Colombini E, Guzzo I, Morolli F, Longo G, Russo C, Lombardi A, et al. Viral load of EBV DNAemia is a predictor of EBV-related post-transplant lymphoproliferative disorders in pediatric renal transplant recipients. Pediatr Nephrol. 2017;32(8):1433-42.

28. San-Juan R, Manuel O, Hirsch HH, Fernández-Ruiz M, LópezMedrano F, Comoli P, et al. Current preventive strategies and management of Epstein-Barr virus-related post-transplant lymphoproliferative disease in solid organ transplantation in Europe. Results of the ESGICH Questionnaire-based Cross-sectional Survey. Clin Microbiol Infect. 2015;21(6):604.e1-9.

29. Savoie BA, Perpbte C, Carpentier L, Joncas J, Alfieri C. Direct correlation between the load of Epstein-Barr virus-infected lymphocytes in the peripheral blood of pediatric transplant patients and. Blood. 1994:2715-22.

30. Kimura H, Ito Y, Suzuki R, Nishiyama Y. Measuring Epstein-Barr virus (EBV) load: the significance and application for each EBVassociated disease. Rev Med Virol. 2008;18(5):305-19.

31. Aalto SM, Juvonen E, Tarkkanen J, Volin L, Haario H, Ruutu T, et al. Epstein-Barr viral load and disease prediction in a large cohort of allogeneic stem cell transplant recipients. Clin Infect Dis. 2007;45(10):1305-9.

32. Gulley ML, Tang W. Using Epstein-Barr viral load assays to diagnose, monitor, and prevent posttransplant lymphoproliferative disorder. Clin Microbiol Rev. 2010;23(2):350-66.

33. Holman CJ, Karger AB, Mullan BD, Brundage RC, Balfour HH Quantitative Epstein-Barr virus shedding and its correlation with the risk of post-transplant lymphoproliferative disorder. Clin Transpl. 2012;26(5):741-7.

34. Van Keerberghen CA, Goffin K, Vergote V, Tousseyn T, Verhoef G, Laenen A, et al. Role of interim and end of treatment positron emission tomography for response assessment and prediction of relapse in posttransplant lymphoproliferative disorder. Acta Oncol (Madr). 2019

35. Zimmermann H, Denecke T, Dreyling MH, Franzius C, Reinke P, Subklewe M, et al. End-of-treatment positron emission tomography after uniform first-line therapy of B-cell posttransplant lymphoproliferative disorder identifies patients at low risk of relapse in the prospective German PTLD registry. Transplantation. 2018;102(5): $868-75$.

Publisher's note Springer Nature remains neutral with regard to jurisdictional claims in published maps and institutional affiliations. 